that we are undergoing with the information and technological revolution we are living through.

\section{FROM VISION ZERO TO PRACTICAL REALITY - KNOWLEDGE TO SHARE}

Matts-Åke Belin. Swedish Transport Administration, Mälardalens University

10.1136/injuryprev-2016-042156.18

Sweden has a long tradition of systematic road safety work and to consider road traffic injuries as a public problem that must be addressed by the national government. This attitude culminated in the Swedish parliament in October 1997 formally adopting Vision Zero as a new long-term goal and direction in road traffic safety work. Vision Zero aims to not only influence directly the concrete work on road safety, but also - more indirectly - the institutional preconditions and approaches, which in turn also have an impact on the actions of various players so that they take action to increase the safety of the road transport system. Vision Zero differs from a more traditional road safety policy with regard to problem formulation, its view on responsibility, its requirements for the safety of road users, and the ultimate objective of road safety work. In this presentation Vision Zero, its implementation and diffusion to other sectors of the society will be presented and discussed.

\section{State of the Art Sessions Tuesday 20.9.2016 13:30-14:30}

\section{Suicide and Self-harm}

\section{SUICIDE PREVENTION: NEED FOR AN INTEGRATED APPROACH}

G Gururaj. Senior Professor and Head, Department of Epidemiology, WHO Collaborating Centre for Injury Prevention and Safety Promotion, Centre for Public Health, National Institute of Mental Health and Neuro Sciences, Bengaluru, India

\subsection{6/injuryprev-2016-042156.19}

Suicide, hitherto a neglected public health problem, has been receiving greater attention in recent years due to growing increase and the accumulating evidence of its preventable nature. Nearly 800000 persons died due to a suicidal act in 2012 as per WHO. Low and Middle Income countries contributed to three fourths of global suicide with significant differences between countries. Age, gender and urban - rural differences are observed between and within countries in suicides.

The spectrum of suicides varies and includes those with suicides, suicidal attempts and suicidal ideations. For every suicide, nearly 10-20 attempt suicide and those with behaviours can only be a guestimate. Suicide is mainly due to a combination of presence of risk factors and absence of protective factors. These can be at an individual, family, societal and system related areas and encompass several biological, social, economic, cultural and environmental factors. The causative factors are often cumulative, repetitive, interactive and prolonged operating on a maladaptive platform.

Global experience has shown that suicides are predictable and preventable in nature. A strong national policy on suicide prevention and/or integrated into other sector's policies and programmes is the need of the hour. Implementation of restricting access to means like control of easy availability of pesticides and drugs, alcohol control policies, positive media reporting, strengthening information systems are urgently required in many countries. Scaling up mental health services for availability and accessibility, destigmatization and decriminalisation will greatly support for reduction of suicides. Many new approaches and strategies are being implemented in several countries that need systematic evaluation.

However, many countries with high rates of suicide have very weak and fragmented policies/programmes along with a lack of good quality and robust data. Intersectoral approaches and skilled human and financial resources are often found lacking. The world Health assembly has set a global target of reducing suicides by $10 \%$ by 2020 and suicide prevention is included under Sustainable Development goals to be achieved by 2030. Achieving these goals and targets needs a strong commitment and enhanced suicide prevention efforts are much needed.

\section{A SUICIDE-PROTECTIVE PAPAGENO EFFECT OF MEDIA PORTRAYALS OF COPING WITH SUICIDALITY}

Thomas Niederkrotenthaler. Associate Professor, Medical University of Vienna, Centre for Public Health, Institute of Social Medicine, Suicide Research Unit

\subsection{6/injuryprev-2016-042156.20}

In addition to an ever-increasing evidence bases of harmful effects of sensationalist reporting on suicide, there is increasing evidence for the positive roles media can play in suicide prevention. For a long time, related research has primarily focused on the question if changing sensationalist media conversation may contribute to the prevention of suicide contagion, so-called Werther effects. Experiences from Austria, the first country worldwide to implement media recommendations back in 1987 as well as from other countries nowadays support that active collaboration with the media can help prevent contagion and improve the quality of reporting.

Changing the media conversation to reporting of suicide prevention is still a different task from actively preventing suicide by media reporting, the so-called Papageno effect. The seminal study on the Papageno effect from 2010 identified an associations between the publication of media reports on mastery of crisis and subsequent decreases in suicide rates in regions where media exposure was strong. Studies using individual data now indirectly support the Papageno effect for different media types. A recent randomised controlled trial indicated that a film featuring an individual who was suicidal but got better mainly because he fell in love (Elizabethtown, USA, 2005) reduced suicide risk factors in the audience. This effect was most pronounced in an audience with increased vulnerability to suicide, suggesting that more suicidal individuals may benefit most from such positive messages. Another randomised controlled trial tested the effects of a newspaper report about an individual who managed to cope with his suicidality by seeking professional help, and showed that the story resulted in a reduction in suicidal cognitions in a subset of participants, but not in a control group (Arendt, Till, \& Niederkrotenthaler, in press).

Most recent findings suggest that also professional online ressources may have a protective effect. In a recent randomised controlled trial (Till, Tran, \& Niederkrotenthaler, 2015) suicide prevention websites featuring professional resources and stories 
of lived experience had a medium-sized impact in terms of a reduction of suicidal ideation in those participants from the general population with baseline suicidality above the sample median. This effect was present immediately after exposure, and was sustained one week later. In particular, personal beliefs about coping skills increased during the trial. Also knowledge related to suicide improved.

Overall, these findings underline that media can make a very relevant contribution to suicide prevention by minimising sensationalist reporting, and maximising reporting on how to cope with suicidality and adverse circumstances. This presentation will review recent progress in research on the Papageno effect, and provide recommendations for future research and practice.

\section{Emergency and Trauma Care}

\section{EMERGENCY CARE SYSTEMS}

Teri Reynolds. Emergency, Trauma and Acute Care, World Health Organisation

\subsection{6/injuryprev-2016-042156.21}

Emergency care is an essential part of the health system and serves as the first point of contact for many around the world. Especially when there are logistical or financial barriers to healthcare access, people may present for care only when symptomatic with acute illness or injury. In most cases, the ill and injured present to frontline providers responsible for the care of both children and adults, with emergencies including injuries, communicable and noncommunicable diseases, and complications of pregnancy. Prioritising an integrated approach to early recognition and resuscitation substantially reduces the morbidity and mortality associated with all of these conditions.

This presentation will review the initiatives of the WHO Emergency, Trauma and Acute Care programme designed to support strengthening of emergency care systems. Techniques for identifying system gaps and for developing planning and funding priorities will be discussed, and the presentation will introduce the WHO Emergency Care System Framework. The Framework captures essential emergency care functions at the scene of injury or illness, during transport, and through to emergency unit and early inpatient care. Different systems may achieve each function in different ways, based on available resources, and the Framework allows policy-makers to use these essential system functions to create context-relevant priority action plans.

\section{Rural and Remote Area Safety}

\section{APPROACHES TO PREVENTION AND CARE OF INJURY IN RURAL AND REMOTE SETTINGS}

Rebecca Ivers. The George Institute for Global Health, Australia

10.1136/injuryprev-2016-042156.22

Traumatic injury is more prevalent in rural and remote settings globally. Large inequalities in the injury burden are found across rural and remote populations of most countries around the world, from the US and Australia to rural China and Bangladesh. Challenges in prevention include over-representation of marginalised populations, fewer preventative programs, implementation gaps and less well developed systems of care, particularly in resource poor settings. These unique challenges call for different approaches.

For decades injury practice and policy has concentrated on a public health approach that identifies burden and risk factors, and develops and implements cost effective programs. However, preventive programs need to incorporate the wide range of risk factors, both structural and individual that contribute to injury, in order to achieve change, and this is challenging to do in a context that focuses on injury as a vertical issue. There are complex interactions between environmental, organisational and personal factors which determine health outside of traditional 'health' services, and systems approaches recognise these, in addition to supporting the critical role of communities in bringing about change. Horizontal programs, that is, those that address systemic issues across disease groups and strengthen health systems maybe more likely to achieve success, especially in remote or resource poor settings where both preventive programs and health services are limited.

The sustainable development goals offer a new opportunity to shift our approach to both injury prevention and care by providing a mechanism to engage multiple stakeholders and work collaboratively. With major goals addressing road injury, universal health care, sustainable transport, disasters, and the rule of law amongst many other goals relevant to injury in rural and remote settings, there are major opportunities to develop cross-cutting programs that prevent injury and improve trauma care and rehabilitation, but also address multiple other conditions. This talk will focus on practical examples of such programs, with case studies from rural and remote settings in both high and low income countries.

\section{AGRICULTURE INJURIES WITH A FOCUS ON RURAL ROADWAY SAFETY: A GLOBAL CHALLENGE}

Corinne Peek-Asa. Associate Dean for Research, College of Public Health; Professor Occupational and Environmental Health; Director, Injury Prevention Research Centre, University of lowa

\subsection{6/injuryprev-2016-042156.23}

Throughout the world, transportation-related injuries are among the leading causes of death for agricultural workers. Rural roadways pose unique risks that contribute to their high fatal crash rate per miles travelled when compared with other types of roads. This presentation will introduce the burden and risk factors for agricultural injuries with a focus on farm equipment safety in different types of global roadway settings. Two case studies will demonstrate the complex factors that contribute to crash risk, ranging from roadway design, to rural population demographics, to traffic safety culture. Effective rural roadway safety requires a multisectoral approach and will require input from all rural roadway users. Prevention and intervention approaches will be discussed within the public health framework, addressing primary, secondary and tertiary prevention and policy, engineering, and educational approaches. 